\title{
Effets de divers modes de fertilisation ( $N, P, K$ ) sur certaines caractéristiques physiques, chimiques, mécaniques et propriétés papetières du pin maritime des Landes (Pinus pinaster Ait.)
}

\section{I. - Caractéristiques physiques, chimiques et mécaniques}

\author{
S. OHTA, R. KELLER * et G. JANIN ** \\ Forestry and Forest Products Research Institute \\ P.O. Box 16, Tsukuba Norin Kenkyu, Danchi Nai, Ibaraki 305, Japon \\ * I.N.R.A., Ecole Nationale du Génie Rural, des Eaux et des Forôts \\ Laboratoire de Recherches sur les Produtits forestiers \\ 14, rue Girardet, $F 54042$ Nancy Cedex \\ *** I.N.R.A., Station de Recherches sur la Qualité des Bois \\ Centre de Recherches forestiêres de Nancy, Champenoux, F 54280 Seichamps
}

\begin{abstract}
Résumé
La qualité du bois d'échantillons de jeunes pins maritimes fertilisés a été étudiée sous divers aspects.

Parmi les sept modalités (témoin, ou apport au semis de $\mathrm{N}, \mathrm{P}, \mathrm{N}+\mathrm{K}, \mathrm{N}+\mathrm{P}, \mathrm{P}+\mathrm{K}$, $N+P+K)$, ce sont les traitements $P+K$ et $P$ qui se sont révélés les plus satisfaisants : ils provoquent une augmentation très sensible de la croissance sans qu'il en résulte une diminution trop importante de la densité du bois ou des propriétés mécaniques mesurées, ce qui est intéressant aussi bien du point de vue de l'utilisation papetière que de celui de la production d'éléments structuraux ì résistance mécanique convenable.
\end{abstract}

\section{Introduction}

Les techniques de ha fertilisation forestière permettent dobtenir des résultats spectaculaires pour la production en volume chez les espèces résineuses susceptibles dune sylviculture intensive.

La littérature étrangère abonde en publications sur ce sujet : elles font le point de l'influence de la fertilisation sur la qualité du bois d'essences résineuses à croissance rapide, par exemple, Parker et al. (1975) pour le douglas, Gintle et al. (1968), RUdman \& MCKinnel (1970 et 1973) pour Pinis radiata. On trouve souvent que la fertilisation provoque une légère diminution de la densité du bois, plus que largement compensée par le gain en volume ; le rendement en fibre peut augmenter et la qualité papetière du bois être améliorée, comme on le trouve dans Siddiquı et al. (op. cit.), et MEgraw \& Nearn (1972). 
Pour l'expérience de Mimizan, sur le pin maritime, Guinaudeau et al. (1963) avaient déjà mis en évidence une action très favorable des engrais, scories de déphosphoration en particulier, sur la croissance, la régularité des pousses en hauteur, la floraison, la durée de vie des aiguilles et la mycorhization sur des arbres semés et fertilisés en 1957.

Par la suite, Polge (1969), étudiant des échantillons provenant de cette même expérience, avait trouvé que la fertilisation provoquait bien une diminution de la densité du bois chez le pin maritime, de l'ordre de 6 à 7 p. 100.

Cette diminution de la densité était d'ailleurs contrebalancée en partie par une augmentation du rendement en fibres pour tous les traitements ayant bénéficié d'un apport de phosphore. Un phénomène analogue est rapporté par SiDDiQur et al. (1972) dans le cas du douglas fertilisé.

De plus, la longueur de fibres des arbres ayant reçu du phosphore avait été trouvée supérieure de presque 9 p. 100 à celle des témoins.

A l'âge de 16 ans, le dispositif de Mimizan avait atteint, selon les traitements, des hauteurs comprises entre 7,20 et $10,72 \mathrm{~m}$; le volume total produit allait de 42,7 à $131,2 \mathrm{~m}^{3}$, soit un accroissement annuel moyen depuis le semis de 2,67 à $8,20 \mathrm{~m}^{3}$ (tabl. 1). Pour les années 1971 et 1972, l'accroissement en volume atteignait de 5,30 à $13,75 \mathrm{~m}^{3 / \mathrm{ha}} /$ an (BONNEAU, 1973, 1974 et 1980 communication personnelle; BonNEAU et al., 1968).

\section{TableaU 1}

Essai de Mimizan.

Hauteur et volume, à la fin de 1972, 16 ans après le début de lexpérience.

Ferilizer experiment at Mimizan.

Total height and volume at the end of 1972, 16 years after the experiment start.

\begin{tabular}{|c|c|c|c|c|c|c|c|}
\hline \multirow{2}{*}{$\begin{array}{l}\text { Caractéristiques } \\
\text { Characteristics }\end{array}$} & \multicolumn{7}{|c|}{$\begin{array}{l}\text { Traitements } \\
\text { Treatments }\end{array}$} \\
\hline & $\mathrm{T}$ & $\mathbf{N}$ & $P$ & NK & NP & PK, & NPK \\
\hline $\begin{array}{l}\text { Hauteur moyenne à la fin de } 1972, \mathrm{~m} . \\
\text { Mean height at the end of } 1972 \text {, in m }\end{array}$ & 7,56 & 7.20 & 10,27 & 7,45 & 10,45 & 10,61 & 10,72 \\
\hline $\begin{array}{l}\text { Volume total produit en } 16 \text { ans, y compris } \\
\text { les coupes d'éclaircies, } \mathrm{m}^{3} / \mathrm{ha} \ldots \ldots \ldots \ldots \\
\text { Total produced volume in } 16 \text { years, in- } \\
\text { cluding thinning crops, in } \mathrm{m}^{3} / \mathrm{ha}\end{array}$ & 51,3 & 42,7 & 122,7 & 51,9 & 128,9 & 131,2 & 130,7 \\
\hline $\begin{array}{l}\text { Accroissement moyen en volume depuis } \\
\text { le semis, pour } 16 \text { ans, } \mathrm{m}^{3} / \mathrm{ha} / \mathrm{an} \ldots . . . \\
\text { Mean volume increment since direct } \\
\text { sowing, in } 16 \text { years, } \mathrm{m}^{3} / \mathrm{ha} / \text { an }\end{array}$ & 3,21 & 2,67 & 7,67 & 3,24 & 8,06 & 8,20 & 8,17 \\
\hline $\begin{array}{l}\text { Accroissement courant en volume pour } \\
1971 \text { et } 1972, \mathrm{~m}^{3} / \mathrm{ha} / \text { an } \ldots \ldots \ldots \ldots \ldots \\
\text { Current annual volume increment for } \\
1971 \text { and } 1972, \mathrm{~m}^{3} / \mathrm{ha} / \text { an }\end{array}$ & 5,90 & 5,30 & 12,80 & 6,00 & 13,55 & 13,75 & 12,40 \\
\hline
\end{tabular}


A un moment où, selon Maugé (1973) et Gelpe \& Maugé (1973) la fertilisation du pin maritime, couramment employée dans tous les reboisements landais au semis et dans les années ultérieures, permet d'escompter une augmentation de la production en volume de plus de 50 p. 100 (production de l'ordre de $10 \mathrm{~m}^{3} / \mathrm{ha} / \mathrm{an}$ ), il est souhaitable de rechercher quelles sont les conséquences de la fertilisation sur cette essence, en particulier sur les propriétés de son bois.

La première partie de cette étude présentera les résultats relatifs à certaines propriétés physiques, chimiques et mécaniques de pins maritimes provenant du dispositif de Mimizan.

La deuxième partie, à paraître ultérieurement, traitera de l'influence de la fertilisation sur les qualités papetières de ces pins en liaison notamment avec la présence de bois de compression.

\section{Propriétés physiques, chimiques et mécaniques}

\subsection{Matériel d'étude}

L'essentiel de l'échantillonnage a été tiré d'arbres âgés de 15 ans, exploités en automne 1971 dans le dispositif de Mimizan.

Il était constitué, en principe, de cinq billons tirés de chacune des cinq répétitions de chaque traitement, soit 25 arbres par traitement. Chaque groupe de cinq billons fut choisi de façon à représenter la variabilité de la circonférence observée dans chaque répétition.

Le dispositif comporte sept traitements :

- témoin (T),

- fertilisation par lazote $(\mathrm{N})$, le phosphore (P),

- fertilisation combinée entre l'azote et le potassium (NK), l'azote et le phosphore (NP), le phosphore et le potassium (PK),

- fertilisation combinéc par les trois éléments (NPK).

Comme le traitement NK n’a pu être représenté que par 22 échantillons, le matériel d'étude comportait 172 billons de un mètre de long, dont la base avait été coupée à cnviron $30 \mathrm{~cm}$ du sol. Les caractéristiques de ces billons sont portées dans le tableau 2.

Un échantillonnage plus restrcint avait été étudié auparavant, à partir d'arbres exploités au printemps 1969 ; il s'agissait d'un arbre par répétition (soit cinq arbres par traitement) dont la circonférence à $1,30 \mathrm{~m}$ était égale à la moyenne des circonférences des arbres de cette répétition. Des mesures de composition chimique en certains éléments, de rétractabilité et d'hétérogénéité du bois ont été faites sur cet échantillonnage réduit.

Les résultats des mesures de largeur de cernes, des propriétés physiques et mécaniques, de la teneur en certains éléments chimiques, de la rétractibilité et de l'hétérogénéité figurent au tableau 3. 


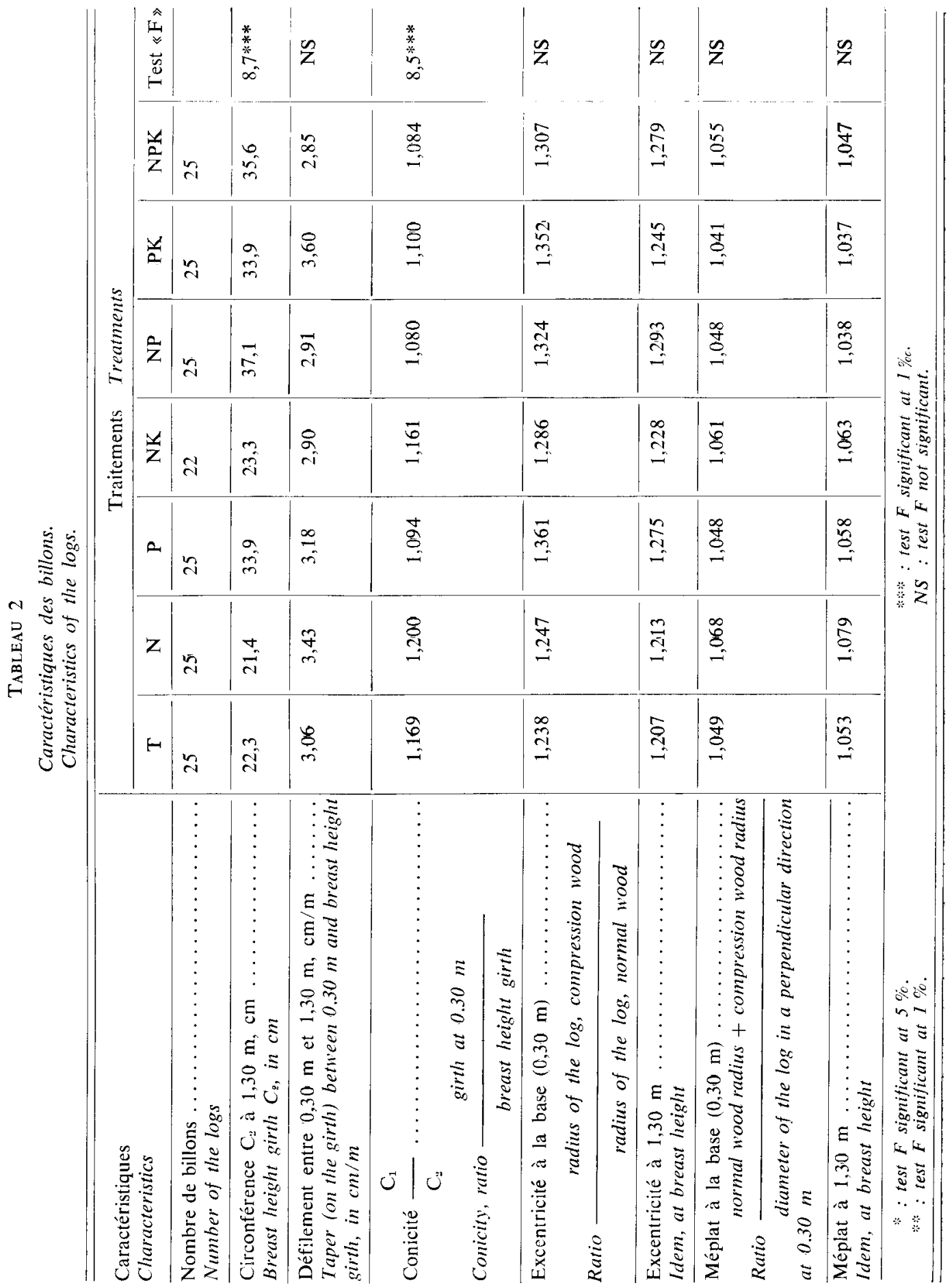


Le tableau 4 regroupe les résultats des tests de comparaisons de moyenne pour lensemble des critères mesurés pour lesquels existent des différences significatives; les traits horizontaux regroupant plusieurs valeurs indiquent qu'elles ne peuvent être tenues pour significativement différentes les unes des autres, aux seuils d'erreur adoptés, 5 p. 100 et 1 p. 100.

\subsection{Etude de la qualité du bois}

\subsection{Caractéristiques des billons}

\subsection{Circonférence à $1,30 \mathrm{~m}$}

L'effet de la fertilisation est manifeste : les traitements comportant du phosphore se différencient fortement des autres, comme l'indique le tableau 2, résultat des comparaisons multiples de moyennes.

Le groupe des traitements $\mathrm{T}, \mathrm{N}$ et $\mathrm{NK}$ ne dépasse pas $23,3 \mathrm{~cm}$ tandis que celui des P, PK, NPK et NP dépasse $33,9 \mathrm{~cm}$, soit au moins une supériorité de $0,7 \mathrm{~cm}$ par an, en moyenne, de la croissance en circonférence pendant les quinze premières années du peuplement.

\subsection{Défilement et conicité}

Aucune différence significative n'a pu être mise en évidence entre les défilements calculés sur les billons de un mètre par l'expression $\frac{\mathrm{C}_{1}-\mathrm{C}_{2}}{1}$, où $\mathrm{C}_{1}$ et $\mathrm{C}_{2}$ sont les circonférences aux extrémités et 1 leur distance.

En revanche, l'examen du rapport $\frac{C_{1}}{C_{2}}$ (1 était très voisin de un mètre pour tous les billons de cette expérience), montre une tendance significative à ce que les billons des traitements sans phosphore présentent une conicité plus marquée que les traitements avec phosphore (tabl. 2).

Toutefois, cette différence de comportement de ces deux variables devrait être réexaminées sur d'autres échantillons pour être confirmée ou infirmée.

\subsection{Excentricité de la moelle}

L'excentricité de la moelle est définie par le rapport du plus grand rayon d'une section transversale au rayon qui lui est directement opposé (Polge \& ILly, 1967); les rayons sont les distances de la moelle aux limites du dernier accroissement annuel.

On observe souvent, dans le cas du pin maritime, une excentricité de la moelle, phénomène lié à la présence de bois de réaction (bois de compression). En première approximation, on peut considérer que le rayon le plus grand se trouve dans la zone de bois de compression et que le plus petit, qui lui est opposé, se trouve dans du bois normal. Cette anisotropie donne une idée du plus ou moins grand développement du bois de compression; si elle est trop accusée elle risque de devenir un défaut gênant, surtout dans l'hypothèse de l'utilisation de cette essence en déroulage. 


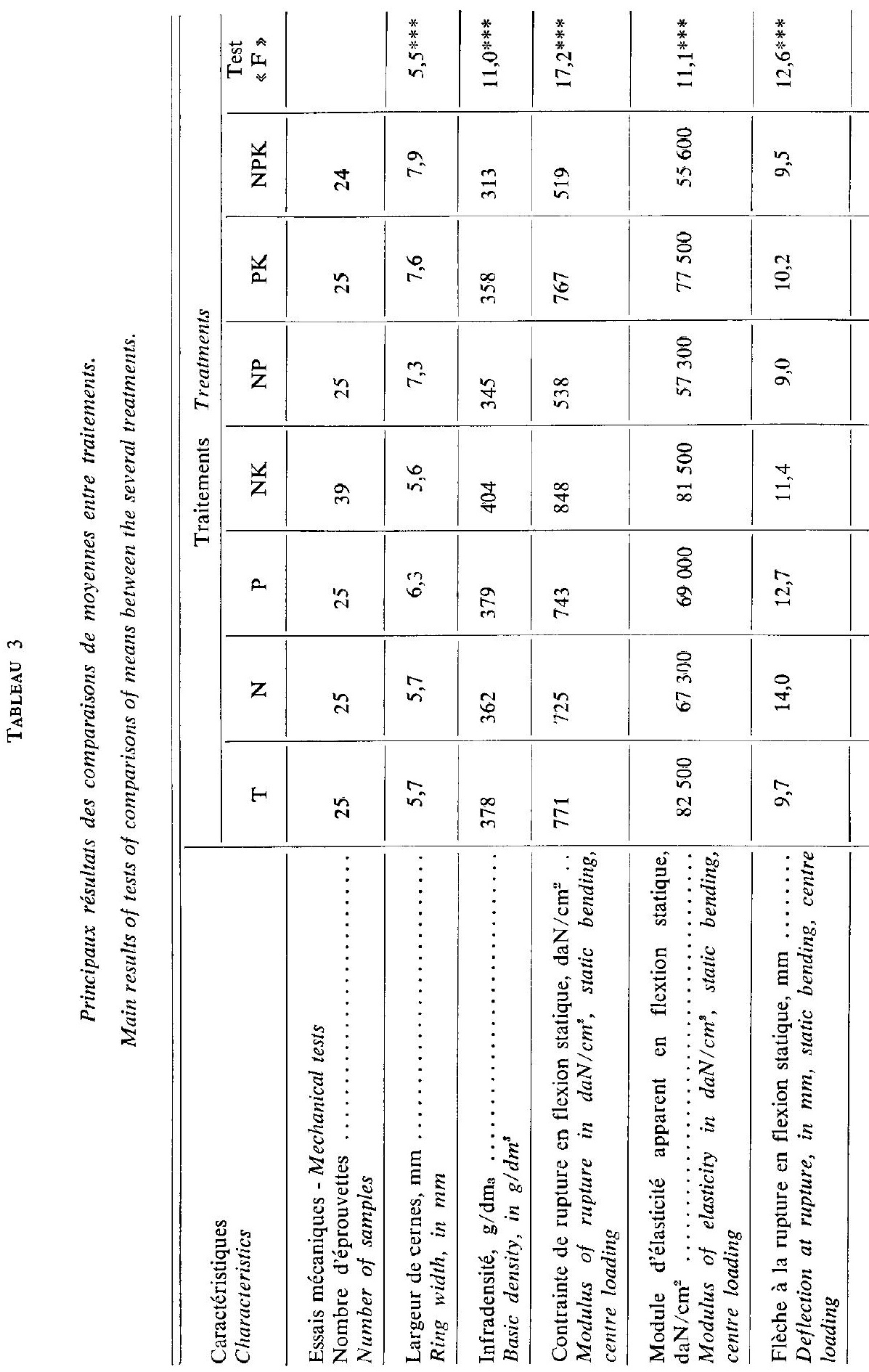




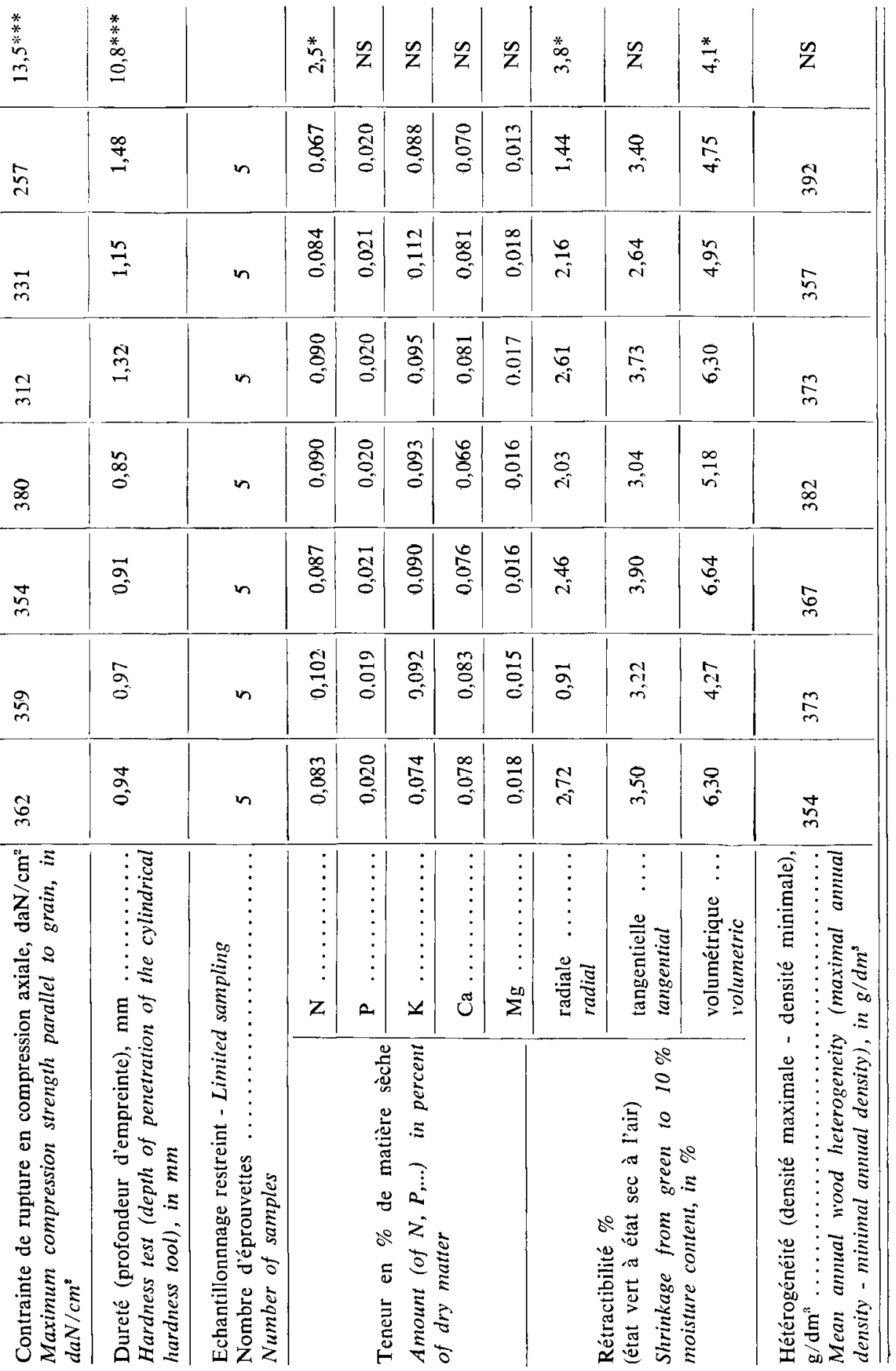


Résultats des comparaisons de moyennes.

Représentation schématique des différences entre traitements

lorsque le test $F$ est significativement différent de zéro.

Results of tests of comparisons of means.

Diagrams of the differences between treatments when the F test is significant.

Several treatments underlined by the same stroke are not statistically different of each others.

Circonférence

$\mathrm{C}_{2}$ à $1,30 \mathrm{~m}$ $\mathrm{cm}$

Conicité $\frac{C_{1}}{C_{2}}$

Excentricité à la base

$(0,30 \mathrm{~m})$

Largeur moyenne des cernes des éprouvettes $\mathrm{mm}$

Infradensité $\mathrm{g} / \mathrm{dm}^{3}$

Contrainte de rupture en flexion statique $\mathrm{daN} / \mathrm{cm}^{2}$

Module d'élasticité apparent en flexion statique $\mathrm{daN} / \mathrm{cm}^{2}$

Flèche à la rupture en flexion statique $\mathrm{mm}$

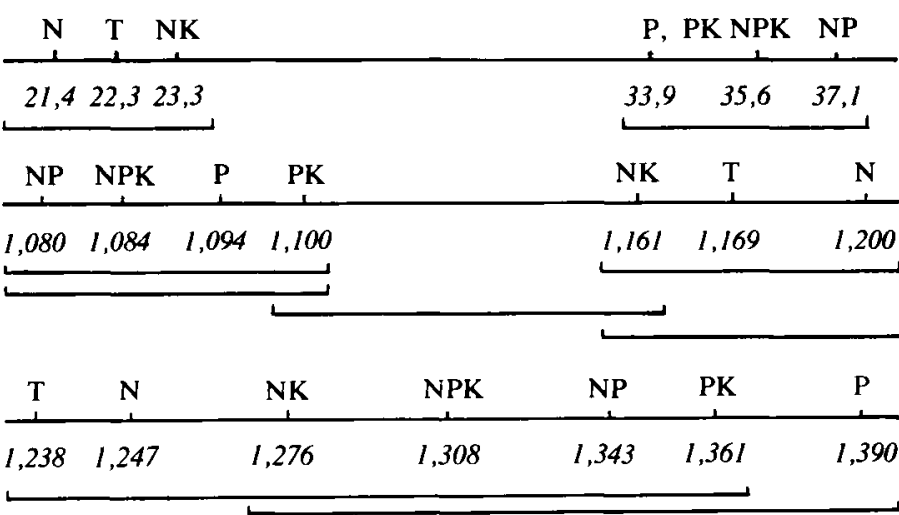
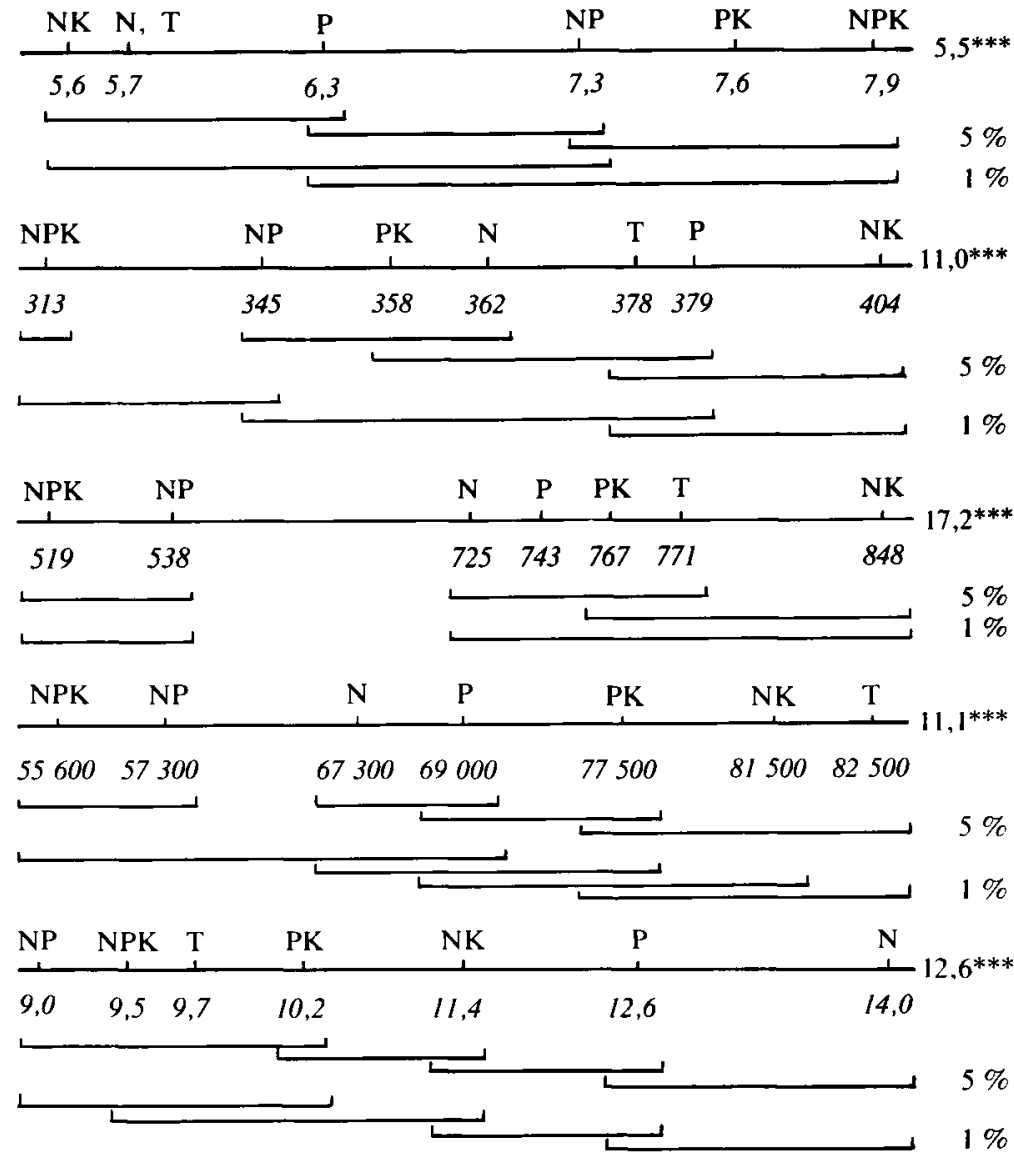
Contrainte de rupture en compression axiale $\mathrm{daN} / \mathrm{cm}^{2}$

Dureté, profondeur d'empreinte $\mathrm{mm}$

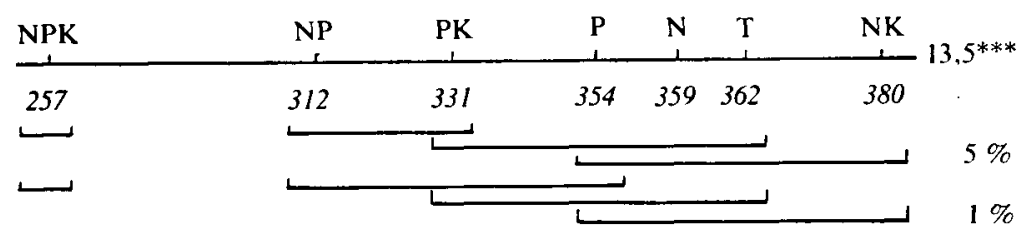

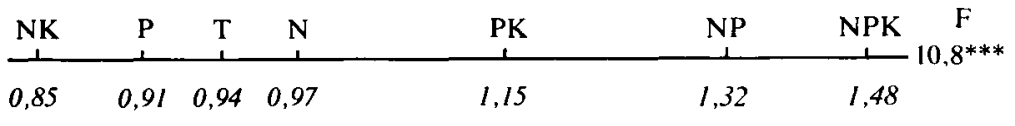

Teneur en azote en p. $100 \mathrm{de}$ matière sèche $N$ content in $p .100$ of dry matter

Teneur en magnésium p. 100 de matière sèche

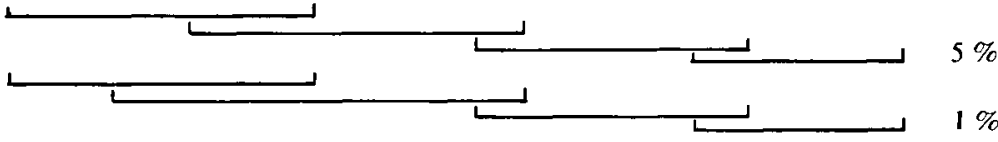

(effet bloc)

Mg content in $p .100$

\begin{tabular}{lcccccc} 
NPK & $\mathrm{T}$ & $\mathrm{PK}$ & $\mathrm{P}$ & $\mathrm{NP}, \mathrm{NK}$ & $\mathrm{N}$ \\
\hline 0,067 & 0,083 & 0,084 & 0,087 & 0,090 & \\
\cline { 1 - 4 } & & & & &
\end{tabular}

of dry matter

( « plot $»$ effect

not "treatment"

effect)

Retrait radial

p. 100

Radial shrinkage, p. 100

bloc $3 \quad$ bloc 2 bloc $4 \quad$ bloc 1 bloc 5

\begin{tabular}{|c|c|c|c|c|c|}
\hline 0,0121 & 0,0151 & 0,0159 & 0,0186 & 0,0187 & \\
\hline 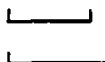 & 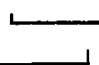 & & & & $5 \%$ \\
\hline
\end{tabular}

Retrait volumétrique p. 100

Volumetric shrinkage, p. 100

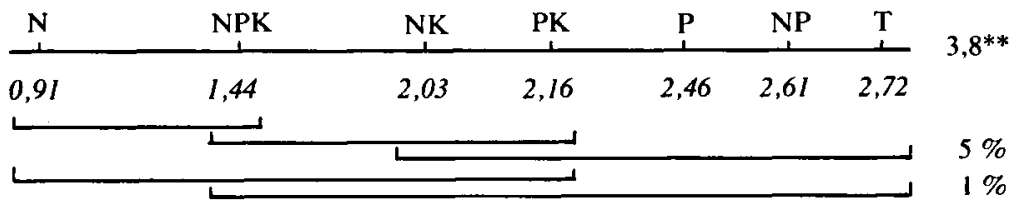

Masse anhydre de bois produite en 16 ans tha

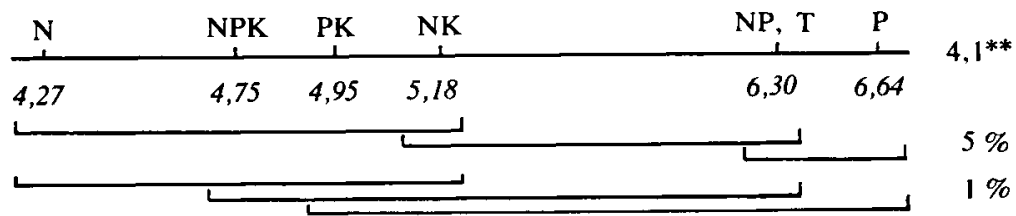

Dry matter weight of wood produced in 16 years,

in tha

\begin{tabular}{|c|c|c|c|c|c|}
\hline $\mathbf{N}$ & $\mathrm{T}$ & NK & NPK & NP & $\mathbf{P}$ \\
\hline 15,5 & 19,4 & 21,0 & 40,9 & 44,5 & 46,5 \\
\hline
\end{tabular}

(1) Test $F$ non calculé, les résultats ayant été obtenus à partir des chiffres moyens de chaque traitement - The $F$ test is not calculated because the results have been obtained from the mean value of each treatment.

Other captions like tables 2 and 3. 
Les chiffres observés (voir le tableau 2) ne permettent pas d'attribuer aux diverses modalités de fertilisation une influence quelconque sur l'excentricité, les tests $\mathbf{F}$ de l'analyse de variance n'ćtant pas significatifs. Compris entre 1,207 et 1,361, avec pour moyenne générale 1,275 , ils se rapprochent des chiffres donnés par PolgE \& ILLY (op. cit.) dont la moyenne est 1,247 .

\subsection{Méplat}

Le méplat a été défini (Polge \& Illy, op. cit.) comme étant le rapport du plus grand diamètre d'une section transversale au diamètre de la direction perpendiculaire.

Comme l'on disposait de la mesure de la circonférence, ainsi que de celle du plus grand diamètre passant par la moelle, on a estimé, de façon approchée, celle du diamètre perpendiculaire en supposant que les sections transversales des billons étaient elliptiques.

A la base des billons, le méplat varie entre 1,041 et 1,068 , mais aucun des traitements ne se distingue des autres, le test $F$ n'étant pas significatif.

A $1,30 \mathrm{~m}$, il fluctue entre 1,037 et 1,079 mais là non plus aucune différence n'apparaît.

Les valeurs obtenues et leur moyenne, 1,053, sont tout à fait comparables aux résultats trouvés par Polge \& Illy (op. cit) pour divers peuplements de pin maritime des Landes, dont la moyenne est 1,039.

\subsection{Caractéristiques des éprouvettes d'essais mécaniques. Résultats}

\subsection{Echantillonnage}

Des éprouvettes normalisées $(2 \mathrm{~cm} \times 2 \mathrm{~cm} \times 30 \mathrm{~cm}$, N.F. B- 51003 à 51008$)$ ont été débitées pour mesurer les propriétés physiques et mécaniques. Nous nous sommes efforcés d'obtenir par traitement au moins 20 éprouvettes utilisables dans les divers essais.

La petite dimension de certains billons, la présence de nombreux nœuds, puisque les arbres étaient encore très jeunes, l'hétérogénéité entre traitements nous ont empêché de disposer d'un nombre donné de billons par traitement et de débiter un nombre donné d'éprouvettes par bilion. L'échantillonnage obtenu est loin d'être orthogonal et, par suite, l'analyse n'est que globale, comme nous l'avons déjà signalé, et porte uniquement sur une influence éventuelle des types de fertilisation, puisqu'on ne peut faire de décomposition de la variance.

\subsection{Largeur de cernes des éprouvettes}

Les différences constatées sur la circonférence des billons se retrouvent, dans l'ensemble, au niveau de la largeur moyenne des cernes des éprouvettes qui varie de $5,7 \mathrm{~mm}$ (traitement témoin $\mathrm{T}$ ) à $7,9 \mathrm{~mm}$ (traitement fertilisé NPK). Le test $\mathrm{F}$ est très significatif (tabl. 4).

La coupure entre traitements avec ou sans phosphore est cependant moins nette que dans le cas de la circonférence (tabl. 2). Cela pourrait être dû au fait que les éprouvettes ont été débitées plutôt dans la partie périphérique des billons, afin d'éviter 
les nouds, et donc dans une zone où la croissance était moins différente de traitement à traitement puisque la période de formation du bois était déjà éloignée de la date d'application des éléments fertilisants.

\subsection{Densité du bois}

La densité a été estiméc par la méthode de la saturation intégrale et les résultats sont exprimés en infradensité (Keyl.werth, 1954 : Polge, 1963). Des différences très significatives apparaissent entre traitements : en particulier le traitement NPK ne présente qu'une valeur de $313 \mathrm{~g} / \mathrm{dm}^{3}$, les autres traitements séchelonnant entre 345 et $404 \mathrm{~g} / \mathrm{dm}^{3}$ (tabl. 3 et 4 ).

Le traitement à $P$ seul a une forte densité, alors que ceux où $P$ est associé à dautres éléments présentent une densité plus faible.

Le traitement PK ne peut être tenu pour significativement différent des traitements $N$, T et $P$, et ceci hien quil ait une largeur moyenne de cernes élevée.

\subsection{Flexion statique}

\subsection{Contrainte de rupture en flexion statique}

Deux traitements présentent des contraintes particulièrement faibles, il sagit de NPK et NP qui forment un groupe bien à part avec des valeurs inférieures à $540 \mathrm{daN} / \mathrm{cm}^{2}$.

Les autres traitements ne sont pas statistiquement différents les uns des autres et l'on peut noter la bonne tenue de PK (tabl. 3 et 4); leurs valeurs sont comprises entre 725 at $848 \mathrm{daN} / \mathrm{cm}^{2}$.

\subsection{Module d'élasticité}

Le module d'élasticité apparent en flexion statique, mesuré à partir des courbes des déformations en fonction de la charge, présente des groupements voisins de ceux de la contrainte de rupture en flexion statique et les traitements sordonnent à peu près comme les valeurs de la densité du bois.

Les modules varient de 55600 à $82500 \mathrm{daN} / \mathrm{cm}^{2}$.

\subsection{Flèche à la rupture en flexion statique} $14 \mathrm{~mm}$.

On trouve un fort domaine de variation puisque les flèches peuvent aller de 9 à

Le classement des traitements nest plus comparable à celui de la densité du bois. ou à ceux des deux grandeurs précédentes : des traitements résistants à une forte contrainte en flexion statique ou encore à densité du bois assez élevée peuvent présenter une faible flèche ( $T$ et $P K$ ) ou, au contraire, fléchir beaucoup ( $P$ et $N$ ).

Il n'a pas été possible ici de mettre en évidence une liaison entre cette flèche et les valeurs de la densité minimale (voir paragraphe 2.233), à la différence de ce qui avait été trouvé dans le cas du douglas (KEller, 1968) pour lequel une corrélation négative entre la flèche et la densité minimale s’était manifestée. 


\subsection{Contrainte de rupture en compression axiale}

Les résultats varient également dans de larges proportions et se classent approximativement comme les densités du bois; ils s"échelonnent de $257 \mathrm{daN} / \mathrm{cm}^{2}$ pour NPK à $380 \mathrm{daN} / \mathrm{cm}^{2}$ pour $\mathrm{NK}$.

\subsection{Dureté}

Les chiffres expriment, en $\mathrm{mm}$, la profondeur de lempreinte laissée par le couteau cylindrique de $3 \mathrm{~cm}$ de diamètre, sous une charge de 200 daN et sur une éprouvette de $2 \mathrm{~cm}$ de côté $(100 \mathrm{daN} / \mathrm{cm})$.

La dureté croît exactement comme les chiffres de densité (le bois est d'autant plus dur que la profondeur d'empreinte est plus faible) ou encore comme la contrainte de rupture en compression axiale.

\subsection{Mesures effectués sur lichantllonnage restreint : composition chimique, rétractibiliti, microdensitométrice}

Le résultats présentés dans les paragraphes 2.231 à 2.233 ont été obtenus à partir de l'échantillonnage restreint tiré de billons exploités en 1969, donc sur des arbres âgés de 13 ans. Ils sont donnés à titre d’information, mais sous réserve des résultats que pourront donner des études portant sur un échantillonnage plus abondant (tabl. 3).

\subsection{Teneur du bois en certains éléments}

On a déterminé la teneur du bois en divers éléments : azote, phosphore, potassium, calcium et magnésium.

En général, les teneurs des divers traitements ne peuvent être tenus pour différentes, sauf peut-être pour l'azote, dont le test $F$ est très proche de la signification au seuil de 5 p. 100 (tabl. 4).

Dans ke cas de cet échantillonnage restreint, la variance a pu être décomposée en un effet «traitement» et un effet «bloc». Les chiffres de la teneur en magnésium ne laissent apparaître aucun effet «traitement » mais, en revanche, un effet «bloc» très significatif dont on peut supposer, en première hypothèse, quiil est provoqué par des différences stationnelles dans le dispositif expérimental (Guinaudeau J. et al., op. cit.). C'est d'ailleurs le scul cas où un effet bloc a pu être mis en évidence à partir de cet échantillonnage restreint.

\subsection{Retractihilité}

Ne seront évoqués ici que les retraits, mesurés sur des cubes de $2 \mathrm{~cm}$ darête, entre le point de saturation des fibres et l'état sec à l'air. soit 10 p. 100 d'humidité environ.

Seuls les retraits radial et volumétrique laissent apparaître des différences significatives entre les traitements. 
Le retrait radial est compris entre 0,91 p. 100 et 2,72 p. 100 et le retrait volumétrique entre 4,27 p. 100 et 6,64 p. 100 (tabl. 4).

Les traitements $\mathrm{N}$ et NPK ont les plus faibles retraits, alors que P, NP ef $T$ sont les plus élevés.

Le retrait axial est compris pour tous les traitements entre $-0,15 \mathrm{p}$. 100 et 0,12 p. 100 et le retrait tangentiel entre 2,64 p. 100 et 3,90 p. 100 , mais aucune différence significative n’a pu être mise en évidence à partir de cet échantillonnage.

\subsection{Microdensitométrie}

Les densités minimale et maximale, ainsi que leur différence qui donne une estimation de l'hétérogénéité du bois (Keller, 1973), ont été mesurées à partir des images radiographiques des six cernes allant de 6 à 11 ans (Pol.ge, 1966; Kri.r.er, 1968).

Aucune différence significative entre traitements n’a pu être mise en évidence pour ces trois caractéristiques.

La densité minimale est comprise entre 351 et $387 \mathrm{~g} / \mathrm{dm}^{3}$; la densité maximale, entre 719 et $752 \mathrm{~g} / \mathrm{dm}^{3}$.

L'hétérogénéité varie de 354 à $392 \mathrm{~g} / \mathrm{dm}^{3}$ selon les traitements.

\section{Conclusion à la première partie}

Pour que la fertilisation ait un intérêt il faut qu'elle augmente la vitesse de croissance sans que la densité du bois et les propriétés mécaniques soient trop fortement diminuées par rapport au traitement témoin.

Les résultats indiquent que les modalités $\mathrm{N}$ et $\mathrm{NK}$ ne sont pas intéressantes puisque la croissance n'est pas meilleure que celle des arbres non fertilisés.

Les traitements NP et NPK, à forte vitesse de croissance, fournissent en revanche le bois le moins dense, aux performances mécaniques très médiocres.

Ils ne seraient intéressants que si, dans l'hypothèse d'une production de masse du bois, le résultat du produit de l'infradensité par le volume élaboré à l'hectare et en un temps donné (par exemple production de matière sèche par hectare et par an) les classait en tête des autres traitements; ce n'est d'ailleurs pas de cas avec nos résultats (tabl. 5). Si l'on a pour objectif la production papetière, le classement doit, en fait, s'appuyer sur le produit : volume $X$ infradensité $X$ rendement en fibres.

Les traitements $\mathbf{P}$ et $\mathrm{PK}$ allient à une très bonne vitesse de croissance, une densité du bois moyenne à forte, et des propriétés mécaniques satisfaisantes, variant de 84 à 91 p. 100 de celles des témoins.

Comme il semble que le retrait de PK soit le plus faible, ce sera, provisoirement, notre meilleur traitement, suivi par $\mathbf{P}$ qui donne aussi la deuxième valeur, après $\mathbf{P K}$, pour la masse de matière sèche (tabl. 5). 


\section{TABLEAU 5}

Masse de bois anlydre produite par chaque traitement pendant les 16 premières années de l'expérience.

Chiffres calculés à partir des résultats moyens.

Dry natter w'ight produced by each treatment during the first 16 years of the experiment.

The figures have been calculated from the mean results.

\begin{tabular}{|c|c|c|c|c|c|c|c|}
\hline \multirow{2}{*}{$\begin{array}{l}\text { Caractéristiques } \\
\text { Characteristics }\end{array}$} & \multicolumn{7}{|c|}{ Traitements } \\
\hline & $\mathrm{T}$ & $\mathbf{N}$ & $\mathrm{P}$ & NK & NP & PK & NPK \\
\hline $\begin{array}{l}\text { Volume total produit en } 16 \text { ans, } \mathrm{m}^{3} / \mathrm{ha} \\
\text { Total volume produced in } 16 \text { years, in } \\
\mathrm{m}^{3} / \mathrm{ha}\end{array}$ & 51,3 & 42,7 & 122,7 & 51,9 & 128,9 & 131,2 & 130,7 \\
\hline $\begin{array}{l}\text { Infradensité, } \mathrm{kg} / \mathrm{m}^{\prime \prime} \ldots \ldots \ldots \ldots \ldots \ldots \\
\text { Basic density, in } \mathrm{kg} / \mathrm{m}^{3}\end{array}$ & 378 & 362 & 379 & 404 & 345 & 358 & 313 \\
\hline $\begin{array}{l}\text { Masse de bois anhydre produite en } 16 \text { ans, } \\
\mathrm{t} / \mathrm{ha} \ldots \ldots \ldots \ldots \ldots \ldots \ldots \ldots \ldots \ldots \ldots \ldots \ldots \ldots \ldots \\
\text { Dry matter weight of wood produced in } \\
16 \text { years, in t/ha }\end{array}$ & 19,4 & 15,5 & 46,5 & 21,0 & 44,5 & 47,0 & 40,9 \\
\hline $\begin{array}{l}\text { Masse de bois anhydre produite annuelle- } \\
\text { ment pendant les } 16 \text { premières années. } \\
\text { t/ha/an ........................... } \\
\text { Dry matter weight of wood annally pro- } \\
\text { duced during the first l } 6 \text { years, in t/ha/an }\end{array}$ & 1,21 & 0,97 & 2,91 & 1,31 & 2,78 & 2,94 & 2,56 \\
\hline
\end{tabular}

Le classement de la modalité avec phosphore seul est particulièrement satisfaisant puisqu'elle correspond à la pratique courante actuelle dans le massif landais : la production est très sensiblement augmentée, la densité du bois et ses propriétés mécaniques sont encore très bonnes; il ny a donc pas lieu de craindre que la sylviculture plus intensive du pin maritime aboutisse à une production de bois de mauvaise qualité.

Il n’en reste pas moins que ces conclusions demandent à être vérifiées par d'autres expérimentations, sur des arbres plus âgés où l'on pourrait étudier aussi l'évolution des propriétés en fonction de l'intervalle de temps séparant la date de fertilisation du pin maritime de la date d’élaboration des différents accroissements annuels.

Reçu pour publication le 11 novembre 1982. 


\section{Sumemary}

Lffects of several fertilizers $(N, P, K)$ on some physical, chemical, mechanical characteristics and pulp propertic's of maritime pine in Landes

(Pinus pinaster Ait.)

\section{Part I : Physical, chemical and mechanical characteristics}

The wood quality of sixten-year-old lertilized maritime pines has been investigated from severit points of view.

Among the seven modalities (control, or fertilization at direct sowing time with $\mathbf{N}, \mathbf{P}$, $\mathbf{N}+\mathbf{K}, \mathbf{N}+\mathbf{P}, \mathbf{P}+\mathbf{K}, \mathbf{N}+\mathbf{P}+\mathrm{K}$ ) the $\mathbf{P}+\mathrm{K}$ and $\mathbf{P}$ treatments have given the best results : they induce a marked increase in yield without a too big drop in wood density or mechanical properties: this fact is interesting in a point of view of production of pulp as well as of structural components with a suitable stiffness.

\section{Zusammenfassung}

Beziehungen zuischen verschiedenen Dünghngsverfahren $(N, P, K)$ und einigen physischen, chemischen, mechanischen Eigenschaften der Se'strandkicfer (Pinus pinaster Ait.) auch in Bezug anf die Zellstoffherstellung

Lirster Teil : Physische, chemische, mechanische Eigenschaften

Holzproben von geclüngten jungen sechzehnjährigen Seestrandkicfern wurden mach verschiedenen Gesischtpunkten untersucht.

Von den folgenden untersuchten Dïngungsverfahren (Kontrol, oder Diingung beim Säen mit $N$. P. $N+K, N+P, P+K, N+P+K$ ) haben sich die zwei Behandlungen mit $\mathbf{P}+\mathrm{K}$ und $\mathbf{P}$ als die besten erwiesen : sie verursachen nämlich eine sehr wichtige Zunahme des Zuwachses ohne eine zu grosse Abnahme der Holzdichte oder der mechanischen Eigenschaften nach sich zu ziehen; dieses Ergebnis ist für die Papierindustrie von Bedeutung sowohl wie fiir die Herstellung von Batuelementen mit passender Festigkeit.

\section{Remerciements}

Nous remercions les Papeteries de Gascogne qui nous ont trìs amablement procuré les échantillons de bois provenant de l'essai de Mimizan-la-Lande ainsi que la Station de recherches sur les sols foresticrs et la fertilisation, en particulier MM. BonNisu et Clément, pour les mesures effectuées et les renseignements donnés.

\section{Références bibliographiques}

Bonnenu M., 1973. La situation de la recherche sur la nutrition des forêts. F.A.O. - I.U.F.R.O. Symposium sur l'ulilisation des engrais en forêt. 3-7 décembre, Paris.

Bonnenu M., 1974. I.es résultats obtenus en France en matière de fertilisation forestière. La Forêt Privée Française, $n^{\circ}$ 99, 67-81.

Bonneau M., Gelpe J., Le Tacon F., 1968. Inflnence des conditions de nutrition minérale sur le dépérissement du pin maritime dans les I andes de Gáscogne. AmI. Sci. l\%or., 25 (4), 251-289). 
Gentle S.W., Bamber R.K., Humphreys F.R., 1968. Effect of two phosphate fertilizers on yield, financial yield, and wood quality of Radiata pine. For. Sci., 14 (3), 283-286.

Guinaudeau J., lely G., Mauge J.P., Dumas F., 1963. Essai de fertilisation minérale sur pin maritime à Mimizan (Landes). Résultats après la $6^{\circ}$ année. Ann. Ec. natl. Eaux Forêts Stu. Rech. Exp., 20, 1-72.

Keller R., 1968. Des caractéristiques nouvelles pour l'étude des propriétés mécaniques des bois : les composantes de la densité. Ann. Sci. for., 25 (4), 237-249.

Keller R., 1973. Caractéristiques du bois de pin maritime. Variabilité et transmission héréditaire. Ann. Sci. for., 30 (1), 31-62.

Keylwerth R., 1954. Eill Beitrag zur qualitativen Zuwachsanalyse. Die Bestimmung der Raumdichtezahl an kleinen Proben. Holz als Roh- und Werkstoff. März, 77-83.

Mavge J.P., 1972. Fertilisation et croissance du pin maritime dans la région landaise. Rapp. annu. A.FO.CEL., 141-175.

Megraw R.A., Nearn W.T., 1972. Detailed D.B.H. density profiles of several trees from Douglas-fir fertilizer/thinning plots. Proceedings of the symposium of the effect of growth acceleration on the properties of wood. For. Prod. Lab. Forest Service U.S.D.A.

Parker M.L., hunt K., Warren W.G., Kennedy R.W., 1975. Effect of thinning and fertilization on intra-ring characteristics and kraftpulp yield of Douglas-fir. Eighth Cellulose Conlerence Syracuse, New York, May, 19-23.

Polge H., 1963. Contribution à l'étude de la qualité du bois des principales essences résineuses exotiques utilisées dans les reboisenments français. Ann. Ec. natl. Eaux Forèts Str. Rech. Exp., 20 (3), 403-467.

Polge H., 1969. Influence de la fertilisation sur la qualité du bois de pin maritime. Ann. Sci. for., 26 (1), 45-64.

Polge H., Illy G.. 1967. Observations sur l'anisotropie du pin maritime des Landes. Ann. Sci. for., 24 (3), 205-231.

Rudman P., McKinnel F.H., 1970. Elfect of fertilizers on wood density of young Radiata pine. Aust. For., 34 (3), December.

Sidnigui K.M., Gladstone W.T., Marton R. 1972. Influence of fertilization on wood and pulp properties of Douglas-fir. Proceedings of the symposium on the effect of growth acceleration on the properties of wood. For. Prod. Lab., Forest Service U.S.D.A. 